\title{
Some periodic and solitary travelling-wave solutions of the short pulse equation
}

\author{
E. J. Parkes ${ }^{\text {a }}$ \\ ${ }^{a}$ Department of Mathematics, University of Strathclyde, Glasgow G1 1XH, UK
}

\begin{abstract}
Exact periodic and solitary travelling-wave solutions of the short pulse equation are derived.
\end{abstract}

Key words: Short pulse equation, periodic travelling-wave, solitary travelling-wave.

PACS: 03.40.Kf

\section{Introduction}

The short pulse equation (SPE), namely

$$
u_{x t}=u+\frac{1}{6}\left(u^{3}\right)_{x x},
$$

models the propagation of ultra-short light pulses in silica optical fibres [1].

In [2] it was shown that the SPE and the sine-Gordon equation (SGE) are equivalent to one another through a chain of transformations. In [3] various known solutions of the SGE were used to generate solutions to the SPE. The kink solution to the SGE leads to a travelling-wave solution of the SPE in the form of a loop soliton. [By a travelling-wave solution, we mean one in which the dependence on $x$ and $t$ is via the single variable $x-v t$, where $v$ is a non-zero constant.] The two-kink and kink-antikink solutions of the SGE also lead to multi-valued solutions of the SPE but they are not travelling waves. The breather solution to the SGE leads to a wave packet solution of the SPE. In the context of light pulses, the latter is the physically relevant solution.

Email address: ejp@maths.strath.ac.uk (E. J. Parkes). 
The aim of the present paper is to complement the work in [3] by presenting other travelling-wave solutions to the SPE. The solution method is similar to the one that we have used previously to find periodic and solitary travelling-wave solutions to the Degasperis-Procesi equation [4], the Camassa-Holm equation [5] and the reduced Ostrovsky equation [6].

In Section 2 we derive an integrated form of the SPE. In Section 3 we find exact one-parameter travelling-wave solutions and classify them. In Section 4 we give our concluding remarks.

\section{An integrated form of Eq. (1.1)}

In order to seek travelling-wave solutions to the SPE it is convenient to introduce new variables $z$ and $\eta$ defined by

$$
z:=\frac{u}{|v|^{1 / 2}}, \quad \eta:=\frac{x-v t-x_{0}}{|v|^{1 / 2}}
$$

where $z$ is an implicit or explicit function of $\eta$, and $v$ and $x_{0}$ are arbitrary constants with $v \neq 0$. In this case (1.1) becomes

$$
\left(z^{2}+2 c\right) z_{\eta \eta}+2 z\left(1+z_{\eta}^{2}\right)=0, \quad \text { where } \quad c:=\frac{v}{|v|}= \pm 1
$$

After one integration (2.2) gives

$$
z_{\zeta}^{2}=f(z)
$$

where

$$
f(z):=B^{2}-\left(z^{2}+2 c\right)^{2}
$$

$B$ is a real positive constant and $\zeta$ is defined by

$$
\frac{d \eta}{d \zeta}=z^{2}+2 c
$$

We note that $(2.2)$ is invariant under the transformation $z \rightarrow-z$. Also, when $c=-1,(2.5)$ indicates that the solutions will have infinite slope when $z^{2}=2$.

\section{Exact travelling-wave solutions of the SPE}

For each choice of $c$, the possible types of travelling-wave solution of the SPE depend on the value, or range of values, of $B$. The types may be classified as follows: 
$3.1 c=1, B>2$

In this case (2.4) may be written

$$
f(z):=\left(a^{2}+z^{2}\right)\left(b^{2}-z^{2}\right), \quad \text { where } \quad a^{2}=B+2, \quad b^{2}=B-2 .
$$

The bounded solutions to (2.2) are such that $-b \leq z \leq b$. By using results 213.00 and 310.02 from [7] to integrate (2.3) and (2.5), we find that these solutions are given in parametric form by

$$
z= \pm \sqrt{\frac{4 m}{(1-2 m)}} \operatorname{cn}(w \mid m), \quad \eta=\frac{[-w+2 E(w \mid m)]}{\sqrt{1-2 m}},
$$

where $w(=2 \zeta / \sqrt{1-2 m})$ is the parameter and

$$
m=\frac{B-2}{2 B} \quad \text { so that } \quad 0<m<\frac{1}{2} .
$$

In $(3.2) \mathrm{cn}(w \mid m)$ is a Jacobian elliptic function and the notation is as used in [8, Chapter 16]; $E(w \mid m)$ is the elliptic integral of the second kind and the notation is as used in [8, Section 17.2.8].

The solution (3.2) is a periodic hump or a periodic well corresponding respectively to the upper or lower choice of sign in (3.2). The wavelength of these solutions is

$$
\lambda=\frac{4|-K(m)+2 E(m)|}{\sqrt{1-2 m}}
$$

where $K(m)$ and $E(m)$ are the complete elliptic integrals of the first and second kind, respectively. Note that $\lambda$ increases from $2 \pi$ to infinity as $m$ increases from zero to 0.5 .

An example of the periodic hump solution is illustrated in Fig. 1.

\section{2 $c=-1, B=2$}

In this case (2.4) may be written

$$
f(z):=z^{2}\left(4-z^{2}\right) .
$$

The bounded solutions to $(2.2)$ are such that $0 \leq z^{2} \leq 4$. Straightforward integration of (2.3) and (2.5) gives

$$
z= \pm 2 \operatorname{sech} w, \quad \eta=-w+2 \tanh w
$$

where $w(=2 \zeta)$ is the parameter. 
The solution (3.5) is either an upright or an inverted solitary loop corresponding respectively to the upper or lower choice of sign in (3.5). The upright solitary loop solution is illustrated in Fig. 2.

With the upper choice of sign, (3.5) is equivalent to [3, equation (13)]. The latter solution was derived from a transformation of the kink solution of the SGE.

\section{$3.3 c=-1,0<B<2$}

In this case (2.4) may be written

$$
f(z):=\left(a^{2}-z^{2}\right)\left(z^{2}-b^{2}\right), \quad \text { where } \quad a^{2}=2+B, \quad b^{2}=2-B .
$$

The bounded solutions to (2.2) are such that $b^{2} \leq z^{2} \leq a^{2}$. By using results 218.00 and 310.02 from [7] to integrate (2.3) and (2.5), we find that these solutions are given in parametric form by

$$
z= \pm \frac{2}{\sqrt{2-m}} \operatorname{dn}(w \mid m), \quad \eta=\frac{[-(2-m) w+2 E(w \mid m)]}{\sqrt{2-m}}
$$

where $w(=2 \zeta / \sqrt{2-m})$ is the parameter and

$$
m=\frac{2 B}{B+2} \quad \text { so that } \quad 0<m<1 .
$$

The solution (3.7) is either periodic upright loops or periodic inverted loops corresponding to the upper or lower choice of sign in (3.7), respectively. The wavelength of these solutions is

$$
\lambda=\frac{2|-(2-m) K(m)+2 E(m)|}{\sqrt{2-m}} .
$$

Note that $\lambda$ increases from zero to infinity as $m$ increases from zero to 1.

An example of the periodic upright loop solution is illustrated in Fig. 3.

In the limit $B \rightarrow 2$ (so that $m \rightarrow 1$ ), (3.7) becomes (3.5), i.e. the periodic loops degenerate to a solitary loop.

$3.4 c=-1, B>2$

In this case (2.4) may be written

$$
f(z):=\left(a^{2}+z^{2}\right)\left(b^{2}-z^{2}\right), \quad \text { where } \quad a^{2}=B-2, \quad b^{2}=B+2 .
$$


The bounded solutions to (2.2) are such that $-b \leq z \leq b$. By using results 213.00 and 310.02 from [7] to integrate (2.3) and (2.5), we find that these solutions are given in parametric form by

$$
z= \pm \sqrt{\frac{4 m}{(2 m-1)}} \operatorname{cn}(w \mid m), \quad \eta=\frac{[-w+2 E(w \mid m)]}{\sqrt{2 m-1}}
$$

where $w(=2 \zeta / \sqrt{2 m-1})$ is the parameter and

$$
m=\frac{B+2}{2 B} \quad \text { so that } \quad \frac{1}{2}<m<1
$$

The solution (3.10) is periodic with wavelength

$$
\lambda=\frac{4|-K(m)+2 E(m)|}{\sqrt{2 m-1}}
$$

Note that $\lambda=0$ at $m=m_{c}=0.826115$ and that $\lambda$ increases from zero to infinity as $m$ decreases from $m_{c}$ to 0.5 , and as $m$ increases from $m_{c}$ to 1 . In the limit $B \rightarrow 2$ (so that $m \rightarrow 1$ ), (3.10) becomes $(3.5)$.

When $m=m_{c}$ the solution (3.10) is a 'figure-of-eight' centered on the origin in the $(\eta, z)$ plane as shown in Fig. 4 . For $0.5<m<m_{c}$ the solution comprises alternating upright and inverted 'bells' whereas for $m_{c}<m<1$ the solution comprises alternating upright and inverted loops. Examples corresponding to the upper choice of sign in (3.10) are illustrated in Figs. 5 and 6, respectively.

For $m=m_{c}$, periodic composite solutions of (2.2) may be constructed. (The notion of composite waves is discussed in [9], for example.) Figure 7 shows the periodic composite solution given in parametric form by

$z=z(w), \quad \eta= \begin{cases}\eta(w)+8 j \eta\left(w_{1}\right), & -w_{1}+4 j K \leq w<w_{1}+4 j K, \\ \eta(w)+(6+8 j) \eta\left(w_{1}\right), & w_{1}+4 j K \leq w<-w_{1}+(2+4 j) K, \\ \eta(w)+(4+8 j) \eta\left(w_{1}\right), & -w_{1}+(2+4 j) K \leq w<w_{1}+(2+4 j) K, \\ \eta(w)+(2+8 j) \eta\left(w_{1}\right), & w_{1}+(2+4 j) K \leq w<-w_{1}+(4+4 j) K,\end{cases}$

where $K:=K\left(m_{c}\right), z(w)$ and $\eta(w)$ are given by (3.10) with the upper choice of sign and $m=m_{c}, w_{1}$ is the root of $z(w)=\sqrt{2}$ such that $0<w_{1}<K$, and $j=0, \pm 1, \pm 2, \ldots$. The wavelength of this solution is $\lambda=8 \eta\left(w_{1}\right)$. The construction of the solution (3.12) is similar to the example in [4, Case 3.3]. 


\section{Concluding remarks}

In Sections 3.1-3.4 we have found exact expressions for travelling-wave solutions to the SPE. For each of the two choices of $c$ these solutions depend on one parameter $B$. There is one type of periodic-wave solution that propagates in the positive $x$ direction. There are several types of periodic-wave solution and one type of solitarywave solution that propagate in the negative $x$-direction; there are places on the wave profile of these solutions where the slope goes infinite. Each solution has a corresponding solution that propagates in the same direction and is a mirror image in the $x$-axis.

\section{References}

[1] Schäfer T, Wayne CE. Propagation of ultra-short optical pulses in cubic nonlinear media. Physica D 2004;196:90-105.

[2] Sakovich A, Sakovich S. The short pulse equation is integrable. J Phys Soc Jpn 2005;74:239-41.

[3] Sakovich A, Sakovich S. Solitary wave solutions of the short pulse equation. J Phys A Math Gen 2006;39:L361-7.

[4] Vakhnenko VO, Parkes EJ. Periodic and solitary-wave solutions of the DegasperisProcesi equation. Chaos Solitons and Fractals 2004;20:1059-73.

[5] Parkes EJ, Vakhnenko VO. Explicit solutions of the Camassa-Holm equation. Chaos Solitons and Fractals 2005;26:1309-16.

[6] Parkes EJ. Explicit solutions to the reduced Ostrovsky equation. Chaos Solitons and Fractals 2007;31:602-10.

[7] Byrd PF, Friedman MD. Handbook of Elliptic Integrals for Engineers and Scientists. Berlin: Springer; 1971.

[8] Abramowitz M, Stegun IA. Handbook of Mathematical Functions. New York: Dover; 1972.

[9] Lenells J. Traveling wave solutions of the Camassa-Holm equation. J Diff Eq 2005;217:393-430. 
List of Figures

Fig. 1.

A typical periodic-hump solution of the SPE with $c=1$ for which $B=4$ so that $m=0.25$ and $\lambda=7.0664$.

Fig. 2

The solitary upright-loop solution of the SPE with $c=-1$ for which $B=2$ so that $m=1$.

Fig. 3.

A typical periodic upright-loop solution of the SPE with $c=-1$ for which $B=1.2$ so that $m=0.75$ and $\lambda=0.48931$.

Fig. 4

The 'figure-of-eight' solution of the SPE with $c=-1$ and $m=m_{c}=0.826115$ so that $B=3.06641$ and $\lambda=0$.

Fig. 5

A typical periodic solution of the SPE with $c=-1$ comprising alternating upright bells and inverted bells for which $B=5$ so that $m=0.7$ and $\lambda=2.58028$.

Fig. 6

A typical periodic solution of the SPE with $c=-1$ comprising alternating upright loops and inverted loops for which $B=2.5$ so that $m=0.9$ and $\lambda=1.64817$.

Fig. 7

The composite periodic solution (3.12) of the SPE with $c=-1$ and $m=m_{c}$ so that $\lambda=5.90747$. 


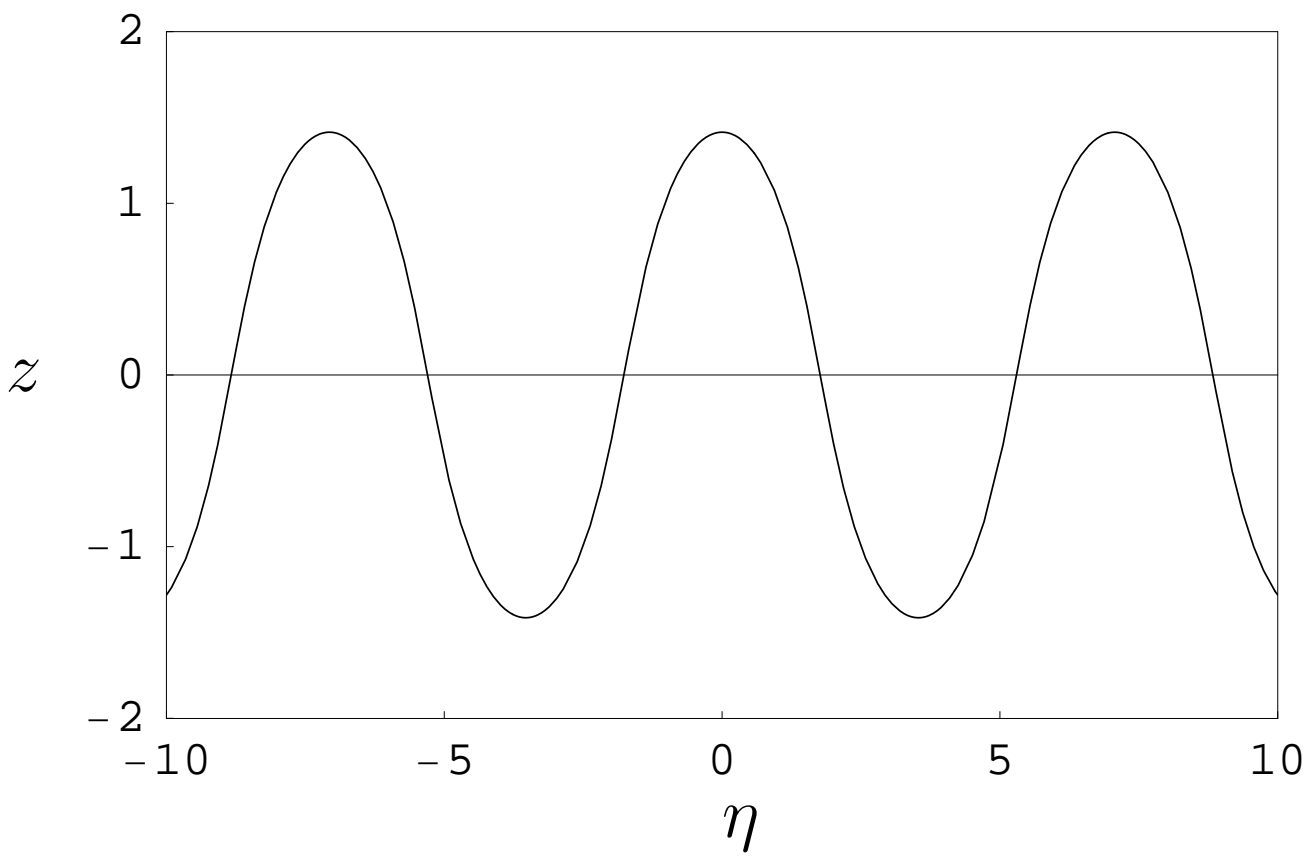

Figure 1 


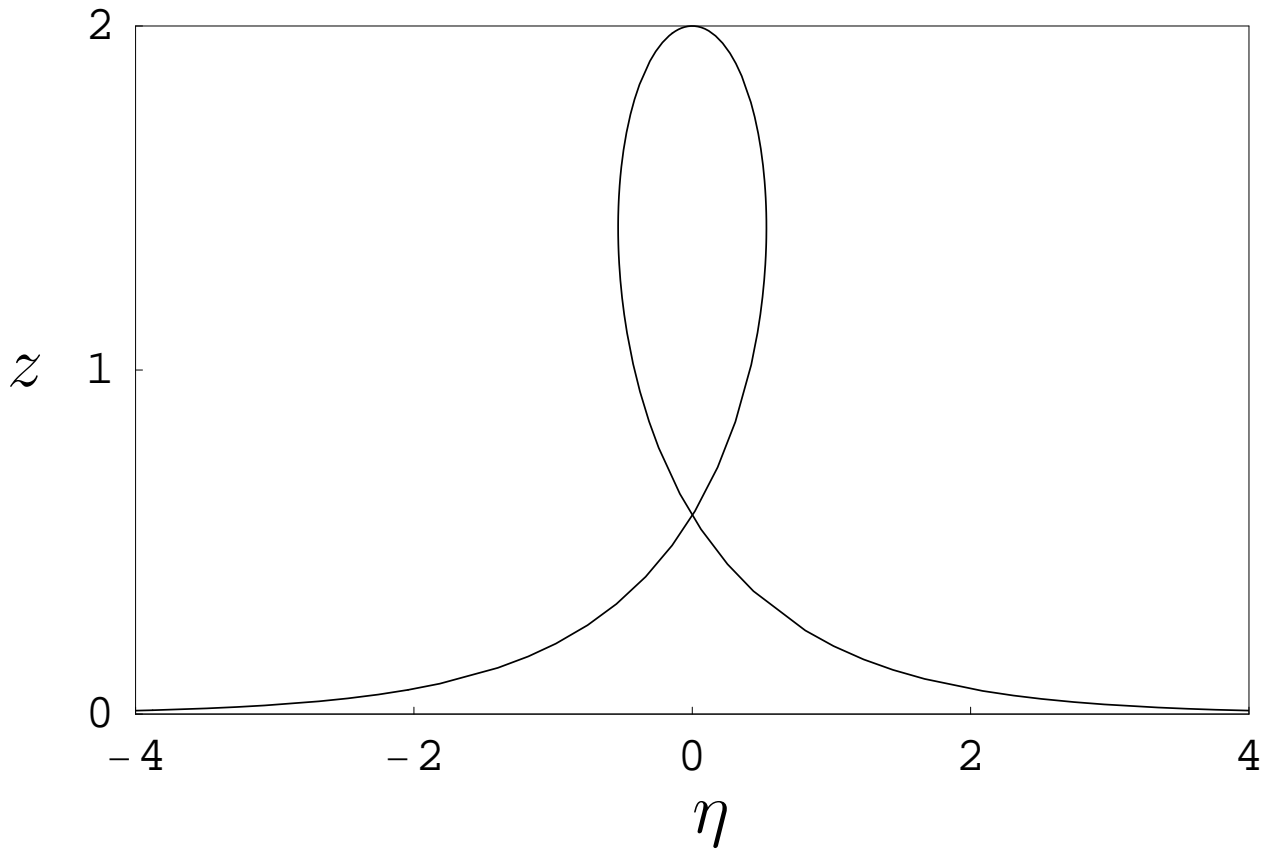

Figure 2 


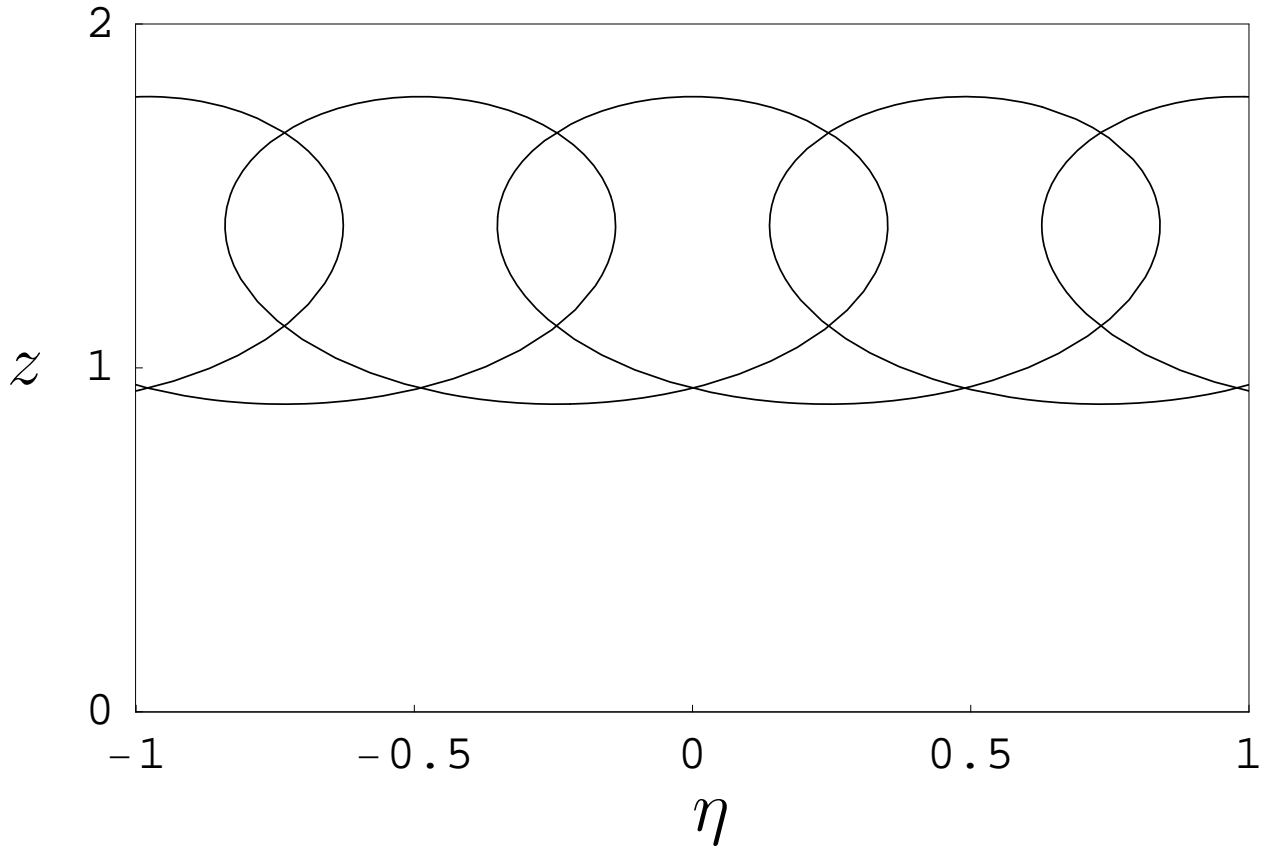

Figure 3 


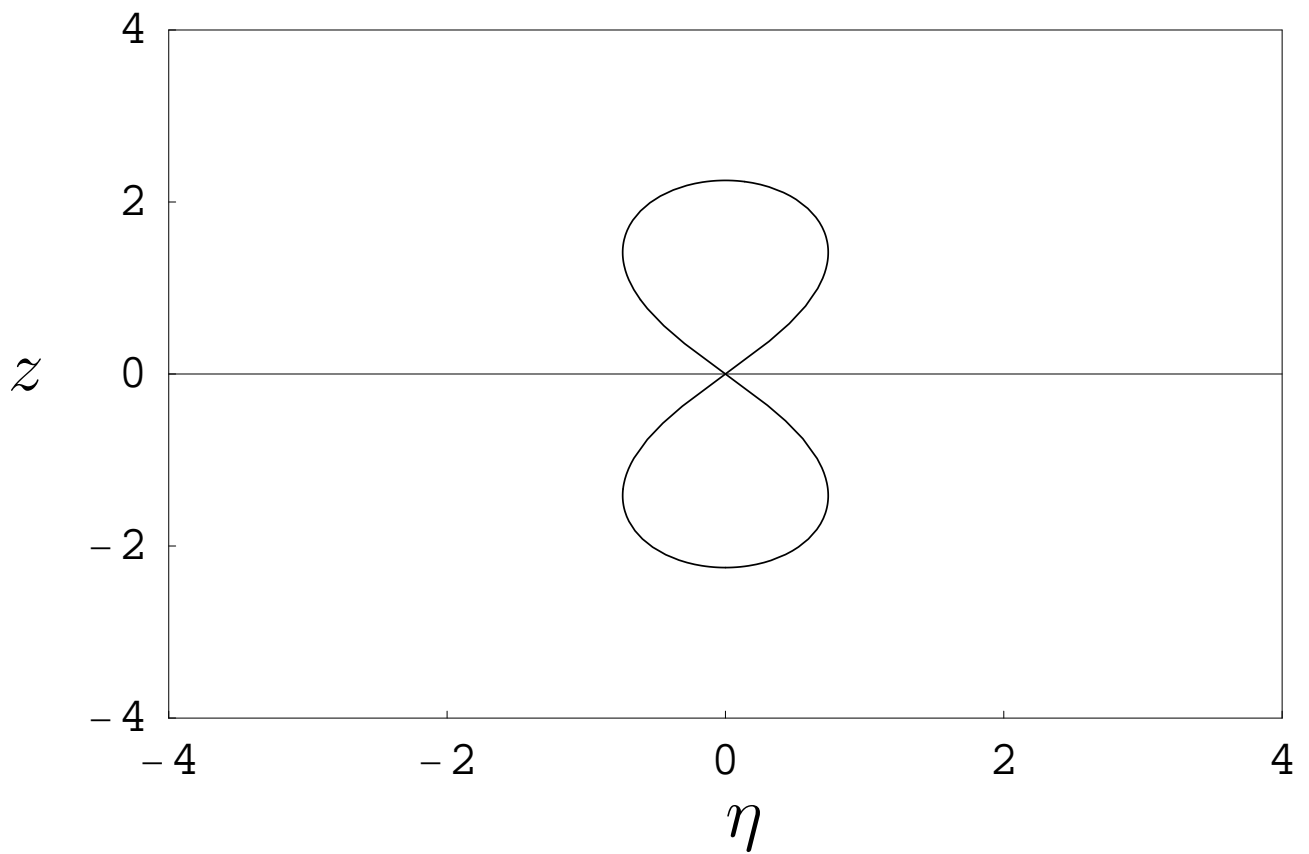

Figure 4 


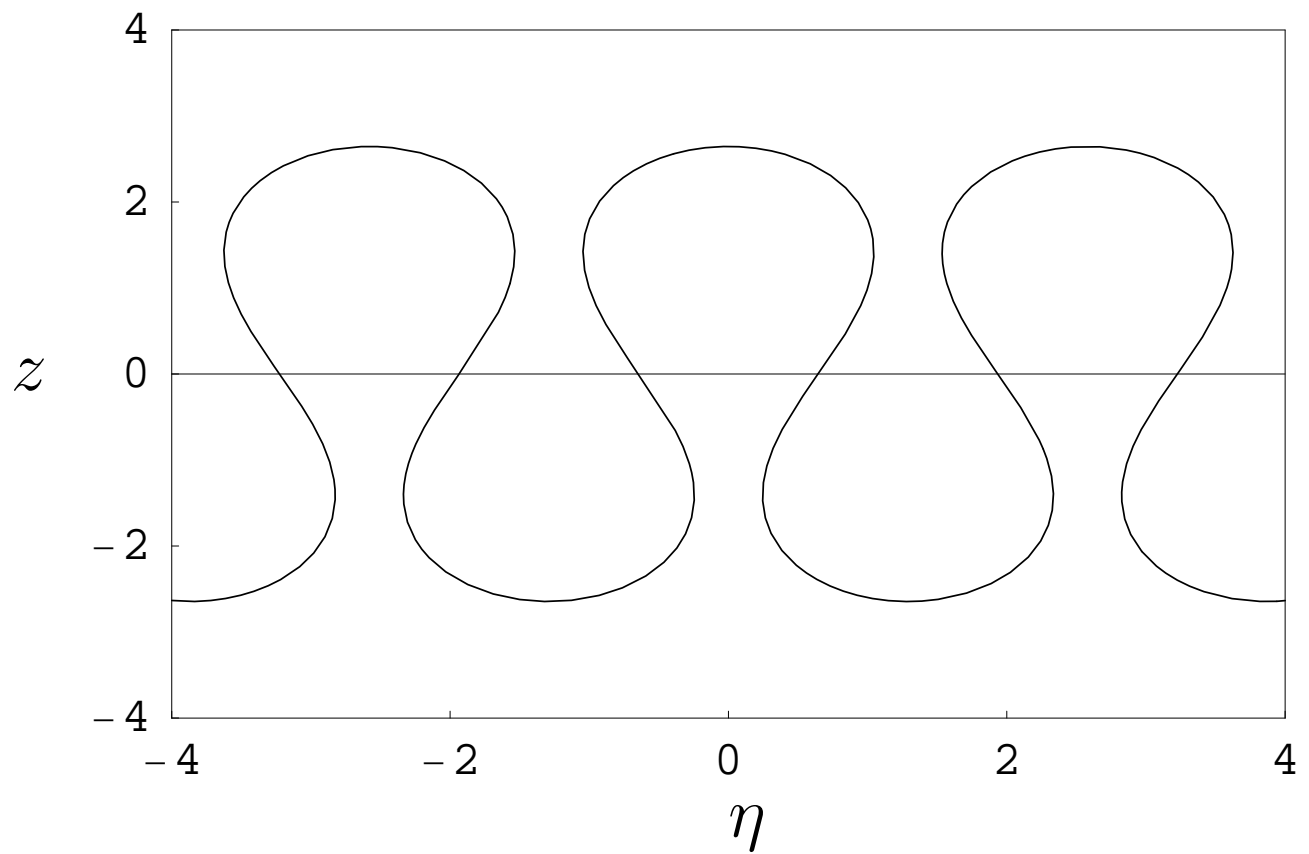

Figure 5 


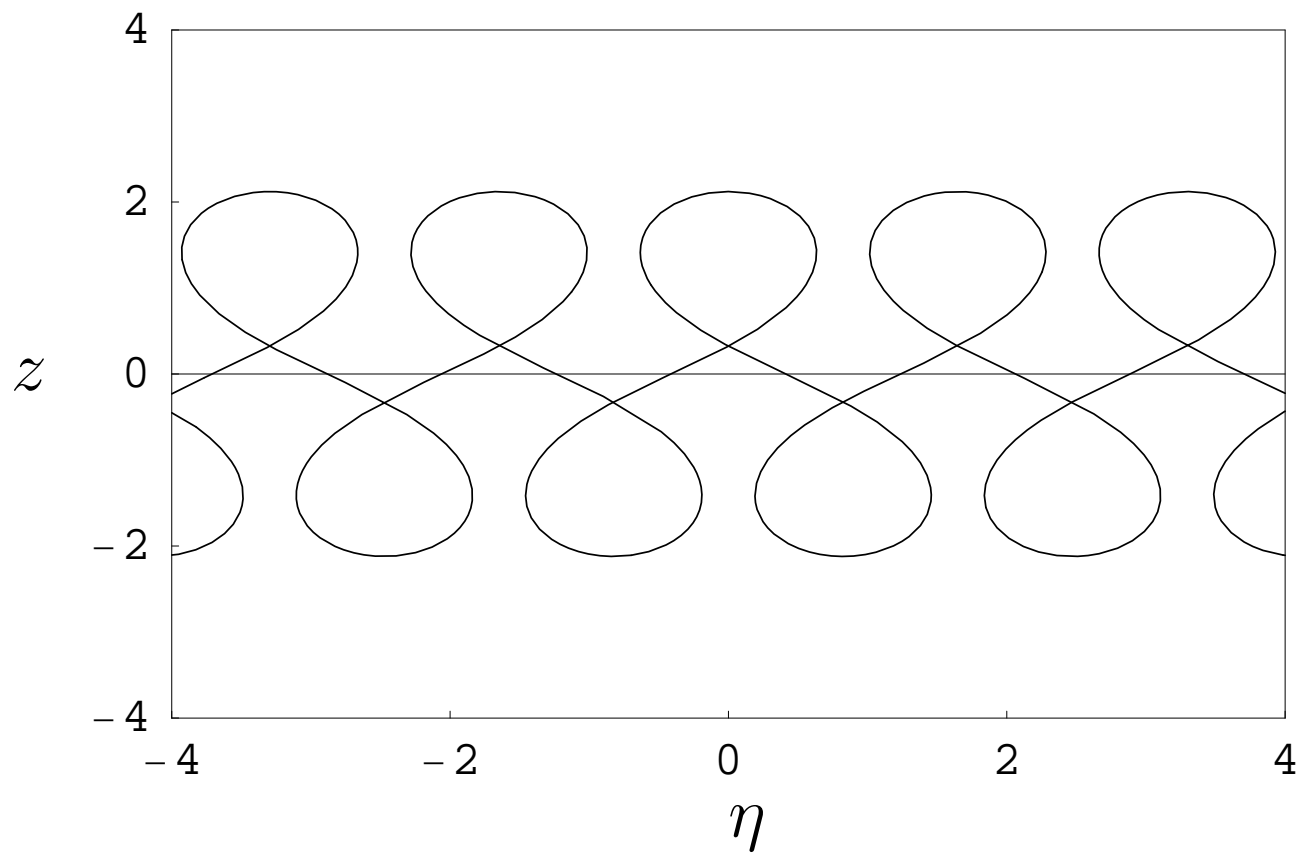

Figure 6 


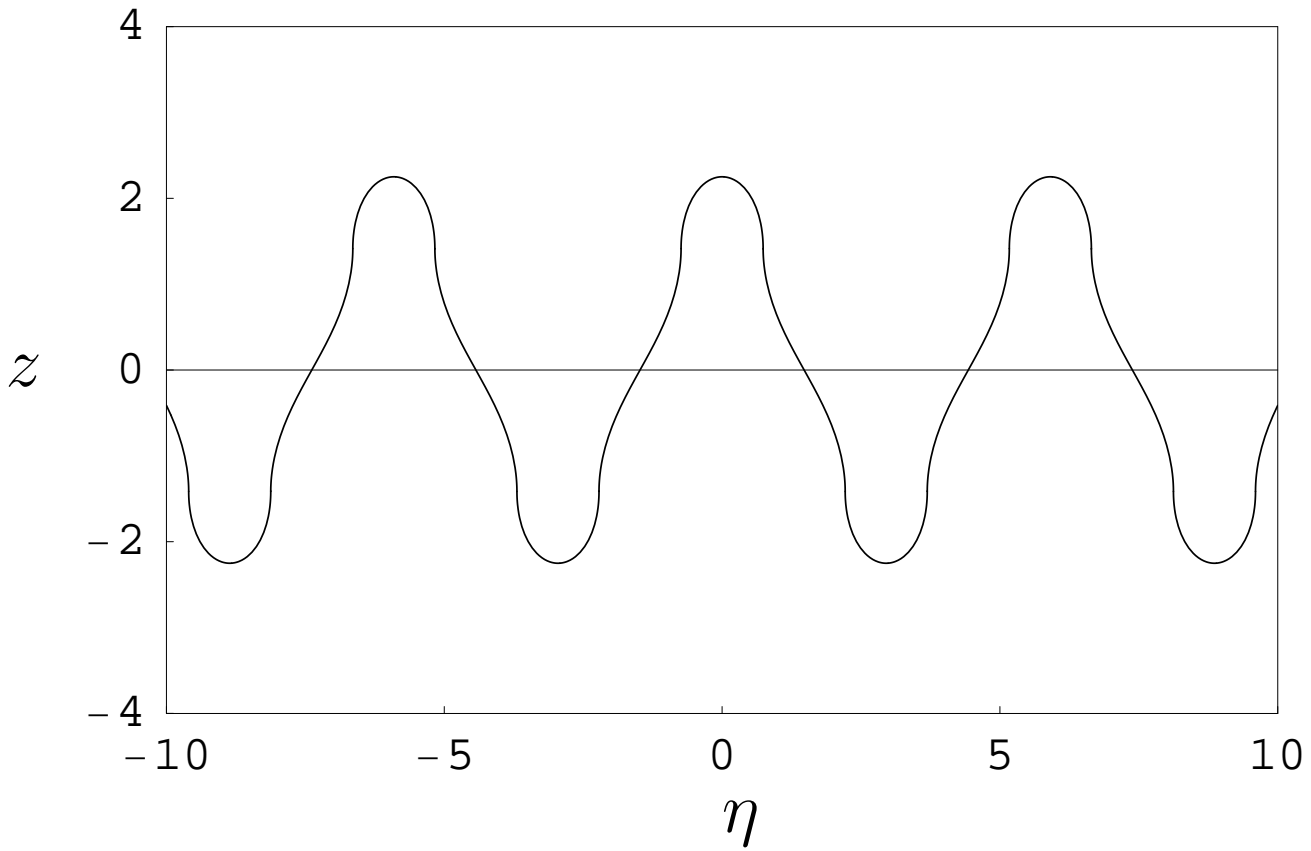

Figure 7 